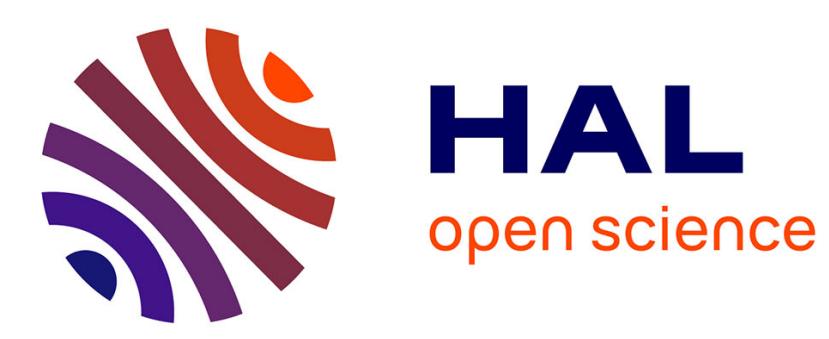

\title{
A homogenization method used to predict the performance of silencers containing parallel splitters
}

Benoit Nennig, Rémy Binois, Emmanuel Perrey-Debain, Nicolas Dauchez

\section{To cite this version:}

Benoit Nennig, Rémy Binois, Emmanuel Perrey-Debain, Nicolas Dauchez. A homogenization method used to predict the performance of silencers containing parallel splitters. Journal of the Acoustical Society of America, 2015, 137 (6), pp.11. 10.1121/1.4921598 . hal-01178969

\section{HAL Id: hal-01178969 \\ https://hal.inria.fr/hal-01178969}

Submitted on 22 Jul 2015

HAL is a multi-disciplinary open access archive for the deposit and dissemination of scientific research documents, whether they are published or not. The documents may come from teaching and research institutions in France or abroad, or from public or private research centers.
L'archive ouverte pluridisciplinaire HAL, est destinée au dépôt et à la diffusion de documents scientifiques de niveau recherche, publiés ou non, émanant des établissements d'enseignement et de recherche français ou étrangers, des laboratoires publics ou privés. 


\title{
A homogenization method used to predict the performance of silencers containing parallel splitters
}

\author{
Benoit Nenniga) and Remy Binois \\ Laboratoire d'Ingénierie des Systèmes Mécaniques et des MAtériaux, (LISMMA-QUARTZ EA2336), \\ SUPMECA, 3 Rue Fernand Hainaut, 93407 Saint-Ouen Cedex, France. \\ Emmanuel Perrey-Debain and Nicolas Dauchez \\ Sorbonne universités, Université de Technologie de Compiègne, \\ Laboratoire Roberval, UMR CNRS 7337, CS 60319, 60203 Compiègne cedex, France.
}

(Dated: July 22, 2015.)

\begin{abstract}
An analytical model based on a homogenization process is used to predict and understand the behavior of finite length splitter/baffle-type silencers inserted axially into a rigid rectangular duct. Such silencers consist of a succession of parallel baffles made of porous material and airways inserted axially into a rigid duct. The pore network of the porous material in the baffle and the larger pores due to the airway can be considered as a double porosity (DP) medium with well-separated pore sizes. This scale separation leads by homogenization to the DP model, widely used in the porous material community. This alternative approach based on a homogenization process sheds physical insight into the attenuation mechanisms taking place in the silencer. Numerical comparisons with a reference method are used to show that the theory provides good results as long as the pressure wave in the silencer airways propagates as a plane wave parallel to the duct axis. The explicit expression of the axial wavenumber in the DP medium is used to derive an explicit expression for the optimal resistivity value of the porous material, ensuring the best dissipation for a given silencer geometry.
\end{abstract}

PACS numbers: 43.20.Mv, 43.50.Gf, 43.28.Py

Keywords: Silencer, Muffler, Baffles, Splitters, Absorbing materials, Porous materials, Effective medium, Double porosity materials, Design

\section{INTRODUCTION}

Baffle and splitter-type silencers are widely used in air conditioning systems in buildings to reduce the noise emitted by air-moving devices such as fans. They consist of a periodic succession of parallel baffles made of porous material (usually mineral wool) and airways inserted axially into a rigid duct acting as a waveguide.

Classical methods used to compute the propagation of acoustic waves through such silencers are generally based on modal techniques, such as the well-known mode matching method. This method relies on solving the appropriate eigenproblem over the cross-section of the duct $^{1-7}$. Eigenfunctions can be solved either analytically $^{5}$, using a root finding algorithm ${ }^{3,6}$ or by discretization techniques using trigonometric function decomposition $^{1,2}$, the finite element method $^{4}$ or layered discretizations $^{7}$. It is also possible to perform threedimensional finite element analysis ${ }^{8,9}$. A comparison between some of these methods can be found in the recent review of $\mathrm{Kirby}^{8}$ on modeling automotive dissipative silencers.

In many practical configurations ${ }^{1,4}$, the frequency range of interest is low enough to allow focusing on the fundamental mode carrying the acoustic energy in the silencer. We will call this type of wave propagation the plane wave regime as the modal profile is almost con-

\footnotetext{
a)Electronic address: benoit.nennig@supmeca.fr
}

stant over the airway cross-section. In this situation, the combination of air and porous layers can be considered as a double porosity medium composed of the pore network of the porous material in the baffle and the larger pores due to the airway. This homogenization approach leads to an explicit expression of the axial wavenumber for the plane wave mode, which greatly facilitates analyzing the performance of this type of silencer. To the authors' knowledge, no attempt has been made to use such an approach for silencer design.

The double porosity (DP) models introduced in audible acoustics by Auriault et $a .^{10}$ and Olny et al. ${ }^{11}$, have been used widely to model the absorption of perforated porous media ${ }^{11-14}$, multiscale porous media such as porous granular beads ${ }^{15}$ and media involving the inclusion of one porous material in another one $\mathrm{e}^{16}$. This concept has given rise to innovations and contributed to the success of heterogeneous materials used for acoustic absorption, especially in the low frequency regime ${ }^{12}$. All these studies were limited to rigid frame porous media ${ }^{17}$ ((see Chap. 5)), though Dazel et al. ${ }^{14}$ has recently proposed a DP model taking into account skeleton elasticity effects.

The main objectives of this paper are: (i) to illustrate the interest and efficiency of DP formalism to model silencers in the plane wave regime; and (ii) to exploit the explicit expression of the wavenumber in the silencer to derive design rules.

The paper is organized as follows. First, the principles of the transfer matrix method (TMM) and the DP model are recalled and combined to obtain a simple baffle-type 


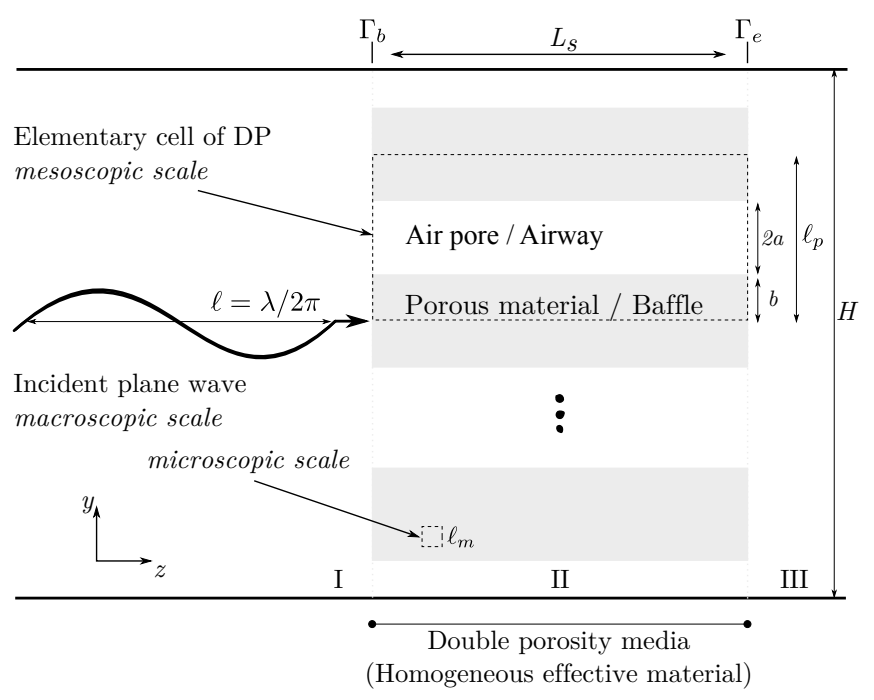

FIG. 1. Geometry of the silencer.

silencer model. The results are shown in terms of Transmission Loss (TL) and compared with reference numerical results associated with the exact geometry of the silencer. Finally, the DP model is used to derive an optimal value for the resistivity yielding the best dissipation for a given silencer geometry.

\section{DOUBLE POROSITY BASED SILENCER MODEL}

\section{A. Problem statement}

The bidimensional silencer considered here and sketched in Fig. 1 consists of a periodic succession of parallel baffles made of porous material and airways. Semiinfinite ducts are present on each side of the silencer (region II), at the inlet (region I) and the outlet (region III). It is assumed that all the regions have the same crosssection $H$ and rigid walls. Let $N$ be the number of baffles, $\phi_{p}=a /(a+b)$ the open area ratio and $\bar{\phi}_{p}=1-\phi_{p}$ its complement, i.e. the filling fraction. These quantities are linked by $H=2(a+b) N$, thus changing the number of baffles implies that the baffle thickness $2 b$ must be modified accordingly ( $H$ and $\phi_{p}$ are kept fixed).

In each region, only the fundamental mode is allowed to propagate, thus the acoustic pressure field $p$ fulfills the one-dimensional Helmholtz equation ( $\left.\mathrm{e}^{\mathrm{i} \omega t}\right)$ along direction $z$ which corresponds to the axial coordinate:

$$
\partial_{z}^{2} p_{i}+k_{i}^{2} p_{i}=0
$$

with wavenumber $k_{i}$. Subscript $i=0$ is used in regions I and III and subscript $i=d p$ in region II. Note that the convection effect is neglected, the Mach number being less than 0.05 for typical buildings applications.

For a simple duct element of length $L$, pressure $p$ and axial velocity $v$ at the beginning (b) of the section are related to pressure $p$ and axial velocity $v$ at its end (e) by

$$
\left(\begin{array}{l}
p \\
v
\end{array}\right)_{b}=\underbrace{\left[\begin{array}{cc}
\cos k_{i} L & \mathrm{i} Z_{i} \sin k_{i} L \\
\left(\mathrm{i} / Z_{i}\right) \sin k_{i} L & \cos k_{i} L
\end{array}\right]}_{\mathbf{T}}\left(\begin{array}{l}
p \\
v
\end{array}\right)_{e}
$$

where $Z_{i}=\rho_{i} c_{i}, \rho_{i}$ and $c_{i}$ are the characteristic impedance, the density and the celerity, respectively, in region $i$. Note that here $i=d p$ as it corresponds to the silencer region. To make the presentation as simple as possible, there are no rigid fairings or perforated plate separating the air domain from the porous media. Under these conditions and for the fundamental mode excitation in region $\mathrm{I}$, the transmission coefficient and the backward reflection coefficient are given by $T=1 / M_{11}$ and $R=M_{21} / M_{11}$, respectively. Here the matrix $\mathbf{M}=\mathbf{B}^{-1} \mathbf{T} \mathbf{B}$, with

$$
\mathbf{B}=\left[\begin{array}{cc}
1 & 1 \\
1 / Z_{0} & -1 / Z_{0}
\end{array}\right],
$$

links the reflected and incident waves. It should be noted that the transfer matrix $\mathbf{T}$ can be modified easily to take into account the presence of a perforated plate on both sides of the silencer, by multiplying $\mathbf{T}$ by the appropriate discontinuity matrices ${ }^{18,19}$. Finally, the transmission loss of the silencer is given by

$$
\mathrm{TL}=-20 \log _{10}|T| .
$$

In principle, the previous analysis only applies below cutoff. In fact, above the cut-off frequency of the first transverse mode $\left(f_{c}=c_{0} /(2 H)\right)$, the analysis still holds as long as the incident, reflected and transmitted pressure fields remain plane. In the present configuration, the periodic and symmetric arrangements of baffles implies that the indexes of the modes propagating through the silencer must satisfy the selection rule ${ }^{1}$ (see (A5) in Appendix A):

$$
n=n^{i n c}+2 q N .
$$

Here, $n^{i n c}$ is the order of the incident mode and $q$ is a relative integer. In the present context, $n^{i n c}=0$ as only the plane wave mode is considered. It follows that when $N=1$, only even modes are allowed to propagate. If $N=3$, only modes of order $n=6 q$ are allowed to propagate, the other modes being forbidden, etc. The selection rules indicates that the wave propagation in the silencer remains in the plane wave regime as long as the frequency is below the cut-off frequency of the mode of order $2 N$.

This propagation model is very simple, but the difficulty now is to determine the effective wavenumber and the characteristic impedance of region II. This is the subject of the next section.

\section{B. Double porosity material}

The acoustics of the double porosity material were established previously by Auriault and Boutin ${ }^{10}$ using periodic structure homogenization techniques. The approach 
was extended by Olny et al. ${ }^{11}$ to clarify the influence, at the macroscale, of contrasting permeability occurring between the macro- and micro-pores of sound absorbing materials. DP models were used to enhance the normal incidence sound absorption of porous materials by Atalla et $a .^{20}$ and Sgard et $a l .{ }^{12}$. In the following, the principles of the DP material model are recalled briefly, using the formalism of Refs. 11, 12, 17 for slit perforations.

Three characteristic lengths are required to describe each scale of the DP material. At macroscopic scale, the characteristic length $\ell$ is governed by the wavelength $\lambda$ such as $\ell \sim \lambda / 2 \pi$. At the mesoscopic scale, $\ell_{p}$ is of the same order as the size of the air gap between the baffle. Finally, at the microscopic scale $\ell_{m}$ is of the same order as the radii of the pores of the porous material. The subscripts $p, m$ and $d p$ are used throughout the paper for the pores (airways), the micropores (porous material) and the double porosity medium (silencer), respectively.

To ensure the separation of scale and apply the periodic structure homogenization method, it is necessary that $\ell_{m} \ll \ell_{p} \ll \ell$. The high permeability contrast assumption will be used throughout this paper. This means that the resistivity of the airway and that of the microporous medium are very different. Therefore, their pore sizes are also different and $\ell_{m} / \ell_{p}<10^{-2}$. This assumption is not restrictive as it allows recovering the low permeability contrast model ${ }^{11}$. The range of validity of the DP model depends on the frequency, the porous material and the geometry.

An additional assumption concerns the length of the silencer $L$ which must be much larger than the mesoscopic length scale $\ell_{p}$ so that both ends of the silencer have negligible effects on the diffusion mechanisms taking place in the double-porosity material. On the basis of these assumptions, Olny et al. ${ }^{11}$ showed that the macroscopic behavior of acoustic waves is given by the Helmholtz equation (1) with the effective wavenumber as follows:

$$
k_{d p}=\omega \sqrt{\rho_{d p} / K_{d p}}
$$

involving an effective density $\rho_{d p}$ and an effective bulk modulus $K_{d p}$. The details of such parameters are now listed for air slits of width $2 a$ between two layers of porous material of width $b$ (the results are also available in Ref. 11 for circular holes). With the dynamic viscosity denoted as $\eta$, the effective density can be written as:

$$
\rho_{d p}=\frac{\eta}{\mathrm{i} \omega \Pi_{d p}}
$$

where $\Pi_{d p}$ is the dynamic permeability in direction $z$ :

$$
\Pi_{d p}=\bar{\phi}_{p} \Pi_{m}+\Pi_{p} .
$$

Here, $\Pi_{m}$ can be deduced from (7) and (B1) by simply replacing the subscript $d p$ by $m$. The effective density $\rho_{m}$ and the effective bulk modulus $K_{m}$ of the porous material are given by the Johnson-Champoux-Allard model, given in appendixB. The dynamic permeability in the mesopore is given by

$$
\Pi_{p}=-\mathrm{i} \phi_{p} \delta_{v}^{2} F\left(\mu_{v}\right)
$$

involving the function

$$
F(\mu)=\left(1-\frac{\tanh \mu \sqrt{\mathrm{i}}}{\mu \sqrt{\mathrm{i}}}\right) .
$$

Here, $\mu_{v}=a / \delta_{v}$ is the ratio between the air gap width and the viscous boundary layer thickness $\delta_{v}=$ $\sqrt{\eta /\left(\rho_{0} \omega\right)}$.

Let us now introduce the bulk modulus in the airways $K_{p}$, obtained using the simplified Lafarge model ${ }^{21}$

$$
K_{p}=\frac{\gamma P_{0} / \phi_{p}}{\gamma-\mathrm{i}(\gamma-1) \frac{\Theta_{p}}{\delta_{t}^{2} \phi_{p}}},
$$

with thermal permeability

$$
\Theta_{p}=-\mathrm{i} \phi_{p} \delta_{t}^{2} F\left(\mu_{t}\right),
$$

the thermal boundary layer thickness $\delta_{t}=\sqrt{\kappa /\left(\rho_{0} C_{p} \omega\right)}$ and the ratio $\mu_{t}=a / \delta_{t}$. Because the viscous and thermal boundary layers are much smaller than the airway area, the quantities $\mu_{t}$ and $\mu_{v}$ are large enough for us to reasonably assume that $F\left(\mu_{t}\right) \approx F\left(\mu_{v}\right) \approx 1$, meaning that

$$
K_{p} \approx \gamma P_{0} / \phi_{p} \text { and } \Pi_{p} \approx-\mathrm{i} \phi_{p} \eta /\left(\rho_{0} \omega\right),
$$

and therefore

$$
\rho_{d p} \approx\left[\frac{\phi_{p}}{\rho_{0}}+\frac{\bar{\phi}_{p}}{\rho_{m}}\right]^{-1} .
$$

The bulk modulus of the DP material is a combination of the bulk modulus of the micro-porous media $K_{m}$ given explicitly in (B2) and $K_{p}$, giving

$$
K_{d p}=\left[\frac{1}{K_{p}}+\bar{\phi}_{p} \frac{F_{d}\left(\omega \frac{P_{0}}{\phi_{m} K_{m}}\right)}{K_{m}}\right]^{-1}
$$

where

$$
F_{d}(\omega)=1-\mathrm{i} \frac{\omega}{\omega_{d}} \frac{D(\omega)}{D(0)},
$$

is a function that links the mean pressure in the micropore (i.e. in the baffle) to the average pressure in the airway. For the slits, Olny et al. ${ }^{11}$ gave $D_{0}=\bar{\phi}_{p} b^{2} / 3$ and

$$
D(\omega)=-\mathrm{i} \bar{\phi}_{p} \delta_{d}^{2} F\left(\mu_{d}\right),
$$

with the ratio $\mu_{d}=b / \delta_{d}$ and the pressure diffusion skin depth $\delta_{d}=\sqrt{\frac{P_{0}}{\sigma \phi_{m} \omega}}$. This parameter gives an estimation of the boundary layer thickness in the material in which strong pressure gradients are taking place. The diffusion frequency is defined as

$$
\omega_{d}=\frac{3 P_{0}}{b^{2} \sigma \phi_{m}},
$$

so that $F_{d}$ has a simpler form quite similar to (10)

$$
F_{d}(\omega)=\frac{\tanh \mu_{d} \sqrt{\mathrm{i}}}{\mu_{d} \sqrt{\mathrm{i}}} .
$$




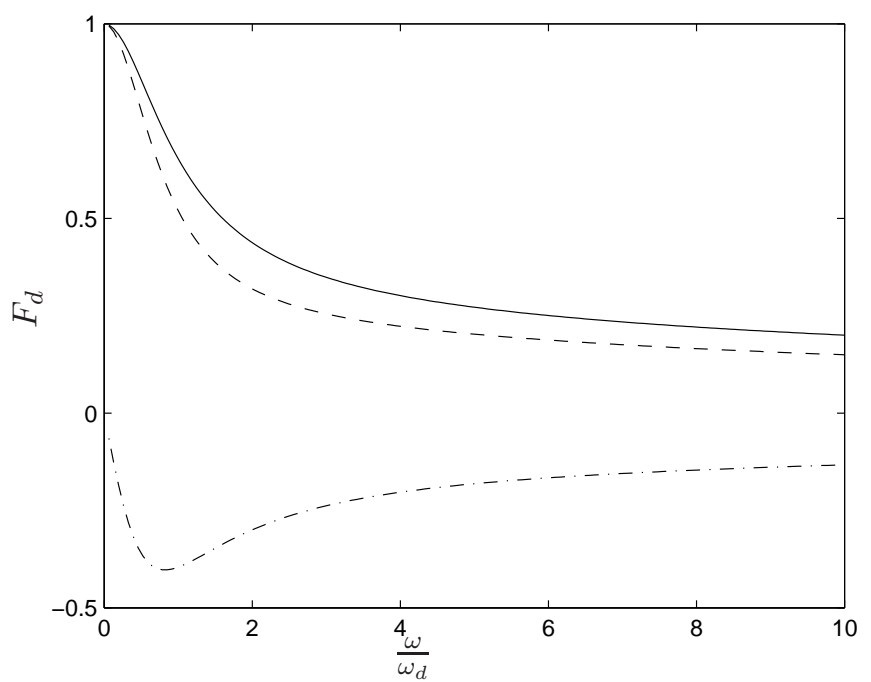

FIG. 2. Evolution of $F_{d}(\omega)$, the ratio between the mean pressure in the micro-porous material and the pore pressure. $-\left|F_{d}\right|,-\cdot-\mathfrak{I m} F_{d},--\mathfrak{R e} F_{d}$ as given in (16).

The imaginary part of this function reaches a minimum when $\omega \approx \omega_{d}$, as shown in Fig. 2 .

Going back to (6) with (7) and (15), it can be shown that the effective wavenumber for the double porosity medium takes the following form:

$$
k_{d p}^{2}=\frac{\phi_{p} \frac{k_{0}^{2}}{\rho_{0}}+\bar{\phi}_{p} F_{d} \frac{k_{m}^{2}}{\rho_{m}}}{\frac{\phi_{p}}{\rho_{0}}+\frac{\bar{\phi}_{p}}{\rho_{m}}} .
$$

Here we have used the fact that the wavenumber in the air is given by $k_{0}=\omega / \sqrt{\gamma P_{0} / \rho_{0}}$. This expression shows that the effective wavenumber resembles a weighted average of the airway wavenumber $k_{0}$ and the micro-porous wavenumber $k_{m}$. This is not an exact rule of mixture due to the function $F_{d}$ which is nearly equal to unity only when $\omega \ll \omega_{d}$.

Before we end this section, it is instructive to observe that function $F_{d}$ can be obtained by another approach. By introducing symmetry arguments, the fundamental mode for the pressure in the material takes the approximate form:

$$
p \approx p_{p} \frac{\cos \left(k_{m} y\right)}{\cos \left(k_{m} b\right)} \mathrm{e}^{-\mathrm{i} \beta z} .
$$

Here, line $y=0$ corresponds to the central axis of the baffle and $p_{p}$ is the air pressure at interface $y=b$. This is a reasonable assumption at a sufficiently low frequency, so it is reasonable to assume that the transverse wavenumber is much larger than the axial one $\beta$, i.e. $k_{m} \gg \beta$. The material behaves as if it were reacting locally while the pore pressure behaves like a forcing term. In these circumstances, it is clear that

$$
\frac{\left\langle p_{m}\right\rangle}{p_{p}} \approx \frac{\tan k_{m} b}{k_{m} b} .
$$

By definition, this quantity is identical to function $F_{d}$ in
(19), thus, after identification:

$$
k_{m} \approx \sqrt{\omega} \cdot \sqrt{-\mathrm{i} \frac{\phi_{m} \sigma}{P_{0}}} .
$$

This expression is precisely the low frequency approximation of the wavenumber in the porous material: $k_{m}=$ $\omega \sqrt{\rho_{m} K_{m}^{-1}}$ (it sufficient to consider the low frequency behavior of $\rho_{m}$ and $K_{m}$ given in the Appendix). The fact that $k_{m}$ increases as the square root of the frequency with the equal real and imaginary parts (remember that $\sqrt{-i}=(1-i) / \sqrt{2})$ is typical of the viscous regime. It corresponds to the low-frequency range: $\omega \ll \omega_{b}$ where

$$
\omega_{b}=\frac{\sigma \phi_{m}}{\rho_{0} \alpha_{\infty}}
$$

is the Biot frequency and when $\omega \approx \omega_{b}$, the inertia forces are of the same order of magnitude as the viscous forces ${ }^{17}$.

\section{Comparison with a reference solution}

In this section the TMM model is compared with a mode matching method developed by the authors in Ref. 7. The model takes into account the existence of evanescent acoustic modes at both ends of the silencer and converges to very accurate solutions as long as the discretization is sufficiently refined. The results are shown for the TL in Fig. 3. The silencer of length $L_{S}=0.3 \mathrm{~m}$ is inserted in a main duct of height $H=$ $0.20 \mathrm{~m}$, and in all cases the airway area is kept constant $\phi_{p}=0.50$ irrespective of the number $N$ of baffles. It is clear that the TMM combined with the DP model (eq. (20)) provides good to excellent agreements, especially in the case of a large number of baffles $(N=5$ here). With wool GW1 of moderate resistivity, the differences never exceed $3 \mathrm{~dB}$. The oscillations in the TL curve at low frequency with wool GW2 and with one baffle resemble those of an expansion chamber. This effect is due to the high resistivity of the micro-porous material (its properties are reported in Tab. I).

With both wools, when $N=1$, discrepancies are very noticeable around $1700 \mathrm{~Hz}$ which corresponds to the cut-off frequency of the first even mode in the rigid duct. When $N=3$ or 5 baffles, the selection rule (A5) indicates that the problem remains essentially one-dimensional even above cut-off. In this regime the DP model remains valid and is expected to produce excellent results over a wide frequency range.

We will now take advantage of the full numerical model $^{7}$ to describe in greater detail the acoustic pressure pattern in the silencer and its correspondence with the diffusion frequency $\omega_{d}$. This frequency separates the behavior of the DP medium into three regimes. In the low frequency regime, i.e. $\omega \ll \omega_{d}$, the pressure is almost uniform over the silencer cross-section. This is illustrated in Fig. 4a when $\omega=\omega_{d} / 3$. When $\omega$ approaches $\omega_{d}$, the wavelength in the micro-porous material is approximately twice the size of the baffle width and the 


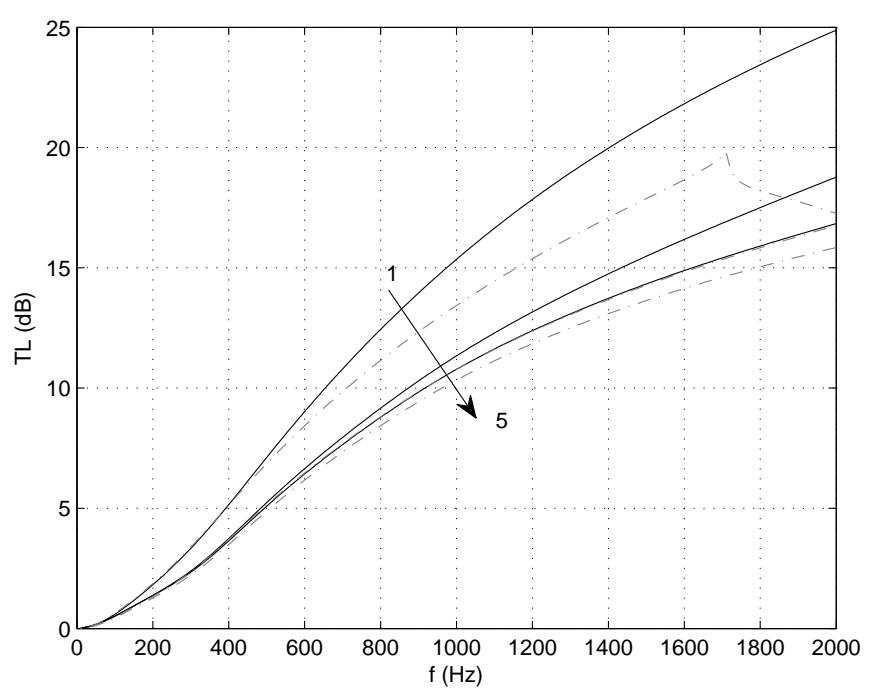

(a) GW1 wool $(\sigma=14066 \mathrm{Nm}-4 \mathrm{~s})$

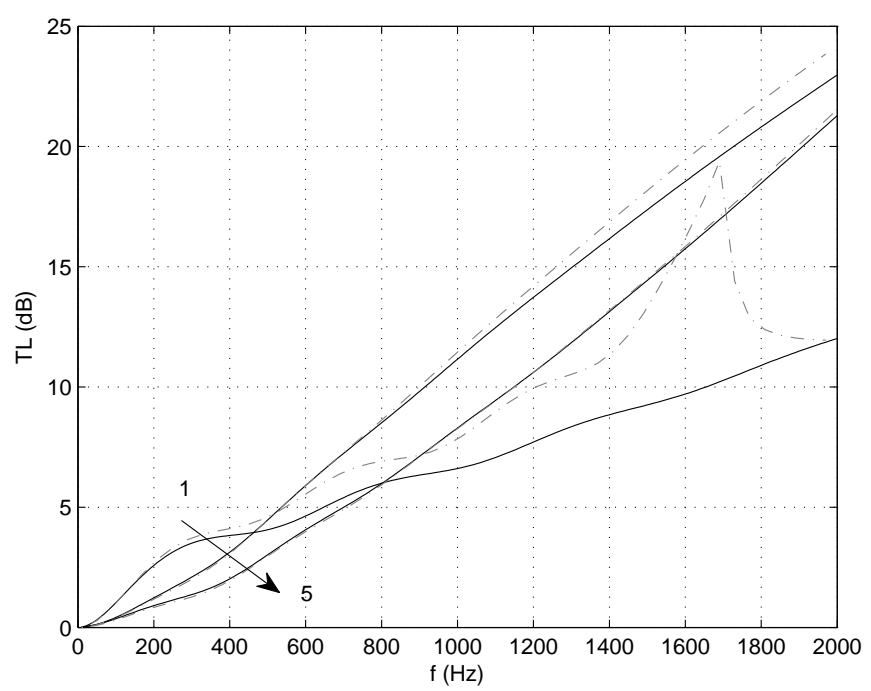

(b) GW2 wool $(\sigma=135000 \mathrm{Nm}-4 \mathrm{~s})$

FIG. 3. Comparison between TL prediction given by a reference $\operatorname{method}^{7}(-\cdot-)$ and the DP model (-) for 1, 3 and 5 baffles in a silencer such $H=0.2 \mathrm{~m}$ and $L_{S}=0.3 \mathrm{~m}$. a) with the GW1 wool, b) with the GW2 wool.

pressure in the core of the baffle is about half the pressure in the airway (see Fig. 4b). Both pore networks (microporous and the airways) are strongly coupled. The pressure fields in each domain present a phase mismatch, generating a new dissipation effect that does not exist in a simple porosity medium ${ }^{11}$, leading to higher attenuation. This effect can be identified in Fig. 3b and for one baffle. In this case, the diffusion frequency $f_{d}=152 \mathrm{~Hz}$ is sufficiently low to be discernible on the TL curve (Table II gives the Biot and diffusion frequencies for different configurations). Finally, when $\omega \gg \omega_{d}$, the pressure vanishes inside the baffle except on a thin boundary layer of thickness $\delta_{d}$ and the core of the baffle has little influ-

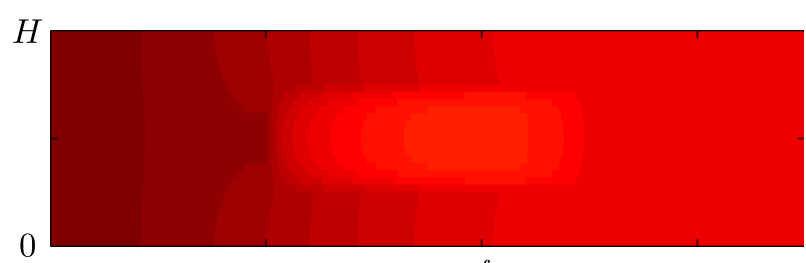

(a) $f=\frac{f_{d}}{3}$

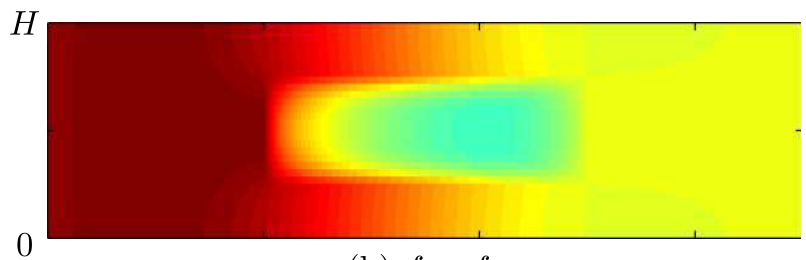

(b) $f=f_{d}$

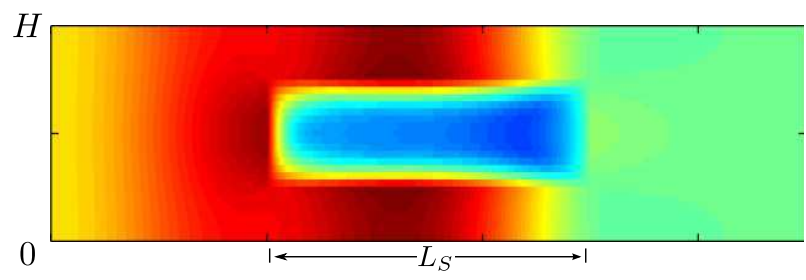

(c) $f=3 f_{d}$

0.

FIG. 4. Normalized pressure field (in absolute value) below, around and above $\omega_{d}$ for a GW2 foam in a silencer such $H=$ $0.2 \mathrm{~m}, L_{S}=0.3 \mathrm{~m}$ and $N=1$. Obtained with a reference solution ${ }^{7}$.

TABLE I. Material properties used in numerical tests. With the porosity $\phi_{m}$, flow resistivity $\sigma$, the tortuosity $\alpha_{\infty}$, the viscous and thermal characteristic lengths $\Lambda$ and $\Lambda^{\prime}$.

\begin{tabular}{ccccccc}
\hline \hline Material & $\phi_{m}$ & $\begin{array}{c}\sigma \\
{\left[\mathrm{Nm}^{-4} \mathrm{~s}\right]}\end{array}$ & $\begin{array}{c}\alpha_{\infty} \\
-\end{array}$ & $\begin{array}{c}\Lambda \\
{[\mu \mathrm{m}]}\end{array}$ & $\begin{array}{c}\Lambda^{\prime} \\
{[\mu \mathrm{m}]}\end{array}$ & $\begin{array}{c}\text { Ref. } \\
-\end{array}$ \\
\hline GW1 & 0.954 & 14066 & 1.0 & 91.2 & 182.4 & {$[7]$} \\
GW2 & 0.94 & 135000 & 2.1 & 49 & 166 & {$[12]$} \\
\hline \hline
\end{tabular}

ence (see Fig. 4c). It can be seen that the pressure inside the airway remains nearly constant over the cross-section area for each case. Knowing $\omega_{d}$ is useful to understand the different dynamical regimes of the silencer as it is strongly linked to its design.

TABLE II. Characteristic frequencies for 1, 3 and 5 baffles with $\phi_{p}=0.5$ and $H=0.2 \mathrm{~m}$.

\begin{tabular}{lcccc}
\hline \hline Material & $f_{b}=\frac{\omega_{b}}{2 \pi}(\mathrm{Hz})$ & \multicolumn{3}{c}{$f_{d}=\frac{\omega_{d}}{2 \pi}(\mathrm{Hz})$} \\
\hline & & 1 & 3 & 5 \\
\cline { 3 - 5 } GW1 & 1760 & 1442 & 12979 & 36053 \\
GW2 & 8000 & 152 & 1372 & 3812 \\
\hline \hline
\end{tabular}




\section{DERIVATION OF OPTIMAL PARAMETERS}

In the previous section the interest of combining the TMM and DP models was shown. This simple approach represented a fast and reliable tool for designing baffle silencers. The aim of this section is to establish guidelines for designing silencers with the best attenuation as a function of the frequency range of interest. Following the one-dimensional analysis, best attenuation is achieved my minimizing the magnitude of the transmitted coefficient

$$
T=\frac{1}{M_{11}}=\frac{1}{\cos \left(k_{d p} L\right)+\mathrm{i} z_{s} \sin \left(k_{d p} L\right)}
$$

where

$$
z_{s}=\frac{1}{2}\left(\frac{Z_{d p}}{Z_{0}}+\frac{Z_{0}}{Z_{d p}}\right)
$$

can be interpreted as an impedance mis-match coefficient for the silencer. The importance of this term depends mainly on the open area ratio $\phi_{p}$ since, in the low frequency limit, it can be shown that

$$
\lim _{\omega \rightarrow 0} \frac{Z_{d p}}{Z_{0}}=\frac{1}{\sqrt{\phi_{p}^{2}+\gamma \phi_{p} \bar{\phi}_{p} \phi_{m}}} .
$$

In practical situations, $25 \%<\phi_{p}<75 \%$ and the magnitude of $Z_{d p}$ never exceeds twice the characteristic impedance $Z_{0}$. Thus, we can reasonably assume that $z_{s}=1+\varepsilon$ where $\varepsilon<0.3$ and consequently

$$
T=\exp \left(-\mathrm{i} k_{d p} L\right)(1+\mathcal{O}(\varepsilon)) .
$$

This signifies that the Transmission Loss is mainly driven by the imaginary part of the wavenumber, i.e. $\mathfrak{I m} k_{d p}$. The explicit expression for the wavenumber $k_{d p}$ in (20), derived on the basis of physical arguments, allows understanding the role of certain parameters, especially the resistivity of the porous material and the open area ratio, which are clearly the most influential. Therefore, it is natural to identify its dependence on these two parameters. In the following sections, the quantity $\mathfrak{I m} k_{d p}$ must be considered as a function of $\omega, \sigma$ and $\phi_{p}$. The dimensionless parameters $r_{\Lambda}$ and $c$ corresponding to the ratio of the characteristic lengths and the square root of the shape factor (see Appendix) respectively, are kept constant in all the calculations, while the thermal and viscous lengths must be considered as functions of $\sigma$ to ensure realistic conditions ${ }^{22}$.

\section{A. Low frequency approximation}

We will assume that the frequency is sufficiently small so that the two dimensionless quantities $\epsilon_{d}=\omega / \omega_{d}$ and $\epsilon_{b}=\omega / \omega_{b}$ are small compared to unity: $\epsilon_{d} \ll 1$ and $\epsilon_{b} \ll 1$. In all cases, low-frequency approximations will be identified by the symbol " ". To the first order, the function $F_{d}$ has the simple form

$$
\tilde{F}_{d}(\omega)=1-\mathrm{i} \epsilon_{d} .
$$

Then using the low frequency approximation of $\tilde{K}_{m}$ given in (B5), we have

$$
\tilde{K}_{d p}=\gamma P_{0}\left[\phi_{p}+\gamma \bar{\phi}_{p} \phi_{m}\left(1-\mathrm{i}\left(\epsilon_{d}+\Upsilon \epsilon_{b}\right)\right)\right]^{-1} .
$$

Finally, replacing the low frequency approximation $\tilde{\rho}_{m}$ in (14) yields the approximated expression for the DP wavenumber:

$$
\tilde{k}_{d p}^{2}=\frac{k_{0}^{2}}{\phi_{p}} \cdot \frac{\phi_{p}+\gamma \bar{\phi}_{p} \phi_{m}\left(1-\mathrm{i}\left(\epsilon_{d}+\Upsilon \epsilon_{b}\right)\right)}{1+\frac{\phi_{m} \bar{\phi}_{p}}{\phi_{p} \alpha_{\infty}} \mathrm{i} \epsilon_{b}} .
$$

A closed form expression for the imaginary part of the DP axial wavenumber can be derived by calculating, to the first order,

$$
\begin{aligned}
& \mathfrak{R e} \tilde{k}_{d p}^{2}=\frac{k_{0}^{2}}{\phi_{p}}\left(\phi_{p}+\gamma \bar{\phi}_{p} \phi_{m}\right), \\
& \mathfrak{I m} \tilde{k}_{d p}^{2}=-k_{0}^{2} \frac{\bar{\phi}_{p} \phi}{\phi_{p}}\left(\epsilon_{d} \gamma+\epsilon_{b}\left(\gamma \Upsilon+\frac{1}{\alpha_{\infty}}+\frac{\gamma \bar{\phi}_{p} \phi_{m}}{\alpha_{\infty} \phi_{p}}\right)\right) .
\end{aligned}
$$

which means that $\mathfrak{I m} \tilde{k}_{d p}$ is of order $\epsilon_{d}$ or $\epsilon_{b}$ and

$$
\mathfrak{I m} \tilde{k}_{d p}=\frac{1}{2} \frac{\mathfrak{I m} \tilde{k}_{d p}^{2}}{\sqrt{\mathfrak{R e} \tilde{k}_{d p}^{2}}}
$$

is a good approximation. In this form, the imaginary part of $\tilde{k}_{d p}$ can be easily differentiated with respect to $\sigma$ and the location of the extremum $\sigma_{0}^{*}$, satisfying $\partial_{\sigma}\left(\mathfrak{I m} \tilde{k}_{d p}\right)\left(\sigma_{0}^{*}\right)=0$ can be found by solving

$$
\frac{\partial \epsilon_{d}}{\partial \sigma} \gamma+\frac{\partial \epsilon_{b}}{\partial \sigma}\left(\gamma \Upsilon+\frac{1}{\alpha_{\infty}}+\frac{\gamma \bar{\phi}_{p} \phi_{m}}{\alpha_{\infty} \phi_{p}}\right)=0 .
$$

The solution to this equation is unique and does not depend on the frequency:

$$
\sigma_{0}^{*}=\sigma_{d b} \sqrt{\left(\frac{1}{\gamma \alpha_{\infty}}+\frac{\bar{\phi}_{p} \phi_{m}}{\phi_{p} \alpha_{\infty}}+\Upsilon\right)} .
$$

The prefactor corresponds to $\sigma_{d b}$, the value taken by the resistivity when the diffusion and Biot frequencies are equal $\left(\omega_{b}=\omega_{d}\right)$,

$$
\sigma_{d b}=\sqrt{\frac{3 P_{0} \rho_{0} \alpha_{\infty}}{b^{2} \phi_{m}^{2}}} .
$$

Physically, this value stems from a compromise between pressure diffusion effects $\left(\omega_{d} \propto 1 / \sigma\right)$ and visco-thermal effects $\left(\omega_{b} \propto \sigma\right)$. It is interesting to evaluate the order of magnitude of each term in the square root of (35) for low tortuosity: $1 / \gamma \approx 0.7$ and $\Upsilon \approx 1$.3. The second term, which involves the ratio between the filling fraction and the open area ratio, is not bounded, although in practice it is reasonable to assume that $1 / 4 \leq \phi_{p} \leq 3 / 4$, meaning that $\frac{\bar{\phi}_{p} \phi_{m}}{\phi_{p}} \in[0.3,3]$. Thus we can already anticipate that in many configurations of practical interest, the optimal value for the resistivity should be chosen in the approximate range: $1.5 \sigma_{d b} \leq \sigma_{0}^{*} \leq 2.5 \sigma_{d b}$. It is noteworthy that this optimal value involves two geometric parameters, the first one, $\phi_{p}$, is dimensionless whereas the height of the baffle $b$ is a real dimension. 


\section{B. Low resistivity and low frequency approximation}

The previous analysis holds as long as the resistivity $\sigma$ is not vanishingly small. Otherwise, $\epsilon_{b}$ cannot be treated as a very small parameter. Although very low resistivity materials (i.e. below $5000 \mathrm{Nm}^{-4} \mathrm{~s}$ ) are seldom encountered in practice, it is interesting to identify the existence of another optimal value for resistivity when both the frequency and resistivity are low. To make the problem tractable, we will set $\epsilon_{d}$ to zero so $F_{d} \approx 1$ and assume that $\epsilon_{b}$ lies somewhere in the region $\epsilon_{b} \in[0.1,1]$. Continuing with these assumptions, closer analysis reveals that the DP wavenumber can be reasonably approximated by

$$
\tilde{k}_{d p}^{2}=k_{0}^{2} \frac{\phi_{p}+\gamma \bar{\phi}_{p} / \phi_{m}}{\phi_{p}+\frac{\bar{\phi}_{p}}{\frac{\alpha_{\infty}}{\phi_{m}}\left(r-\mathrm{i} \frac{\omega_{b}}{\omega}\right)}} .
$$

The extremum problem $\partial_{\sigma}\left(\mathfrak{I m} \tilde{k}_{d p}\right)=0$ using (33) can be resolved analytically and we find that the solution depends linearly on the frequency as

$$
\sigma_{1}^{*}(\omega)=\omega \cdot \frac{\rho_{0} \alpha_{\infty}}{\phi_{m} \phi_{p}}\left(r \phi_{p}\right)^{\frac{1}{4}}\left(\frac{\phi_{m} \bar{\phi}_{p}}{\alpha_{\infty}}+r \phi_{p}\right)^{\frac{3}{4}} .
$$

Unlike the previous case, the thickness of the baffle $b$ is not involved and formula (38) remains unchanged if the number of baffles is modified while keeping $\phi_{p}$ constant.

\section{Discussion}

\section{Validity of Eqs. (35) and (38)}

The case of a typical baffle silencer is now analyzed and discussed for 3 values of the open area fraction $\phi_{p}: 0.25$, 0.5 and 0.75 . In every case, the silencer is composed of one baffle based on GW1 wool $\left(r_{\Lambda}, c, \alpha_{\infty}, \phi_{m}\right)$ and the height of the duct is $H=0.2 \mathrm{~m}$. In Figs. 5, the attenuation rate in the DP material, i.e. the imaginary part of the wavenumber $k_{d p}$ normalized by $k_{0}$, is plotted as a function of the frequency and resistivity. It should be noted that the best acoustic attenuations occur in the white area, the extent of which depends strongly on the open area ratio and, as expected, the larger value of $\phi_{p}$ is, the worse the silencer attenuation.

The maximum (with respect to $\sigma$ ) attenuation rate given by the DP model is also shown and these optimal values are compared with those calculated with the reference model $^{7}$. For the latter, the axial wavenumber $\beta$ corresponding to the fundamental mode is considered. The locations of these maximums are in good agreement though discrepancies may occur in regions where $\mathfrak{I m} k_{d p}$ is nearly constant with respect to the resistivity. When this happens, a jump from one isoline to the other can be observed, as shown in Fig. 6. The frequency of this jump depends mainly on the open area ratio. At low frequency, the best attenuation closely follows the theoretical predictions given by $\sigma_{1}^{*}(\omega)$ in (38) and above the jump frequency the value for the resistivity $\sigma_{0}^{*}$ in (35) can be observed to be nearly optimal.

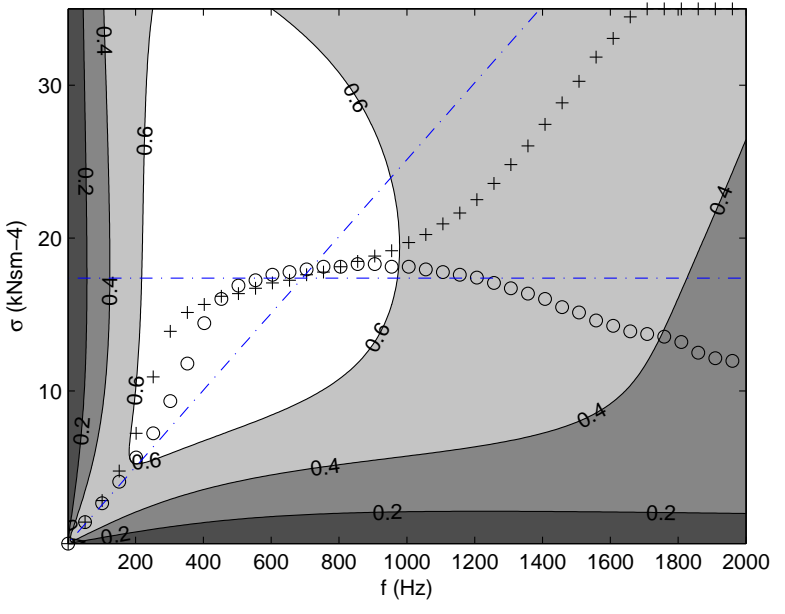

(a) $\phi_{p}=0.25$

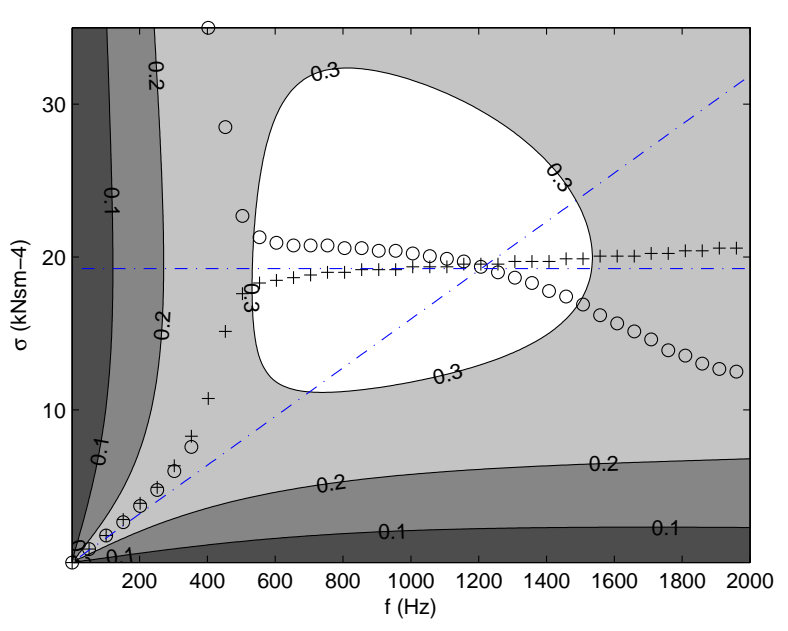

(b) $\phi_{p}=0.50$

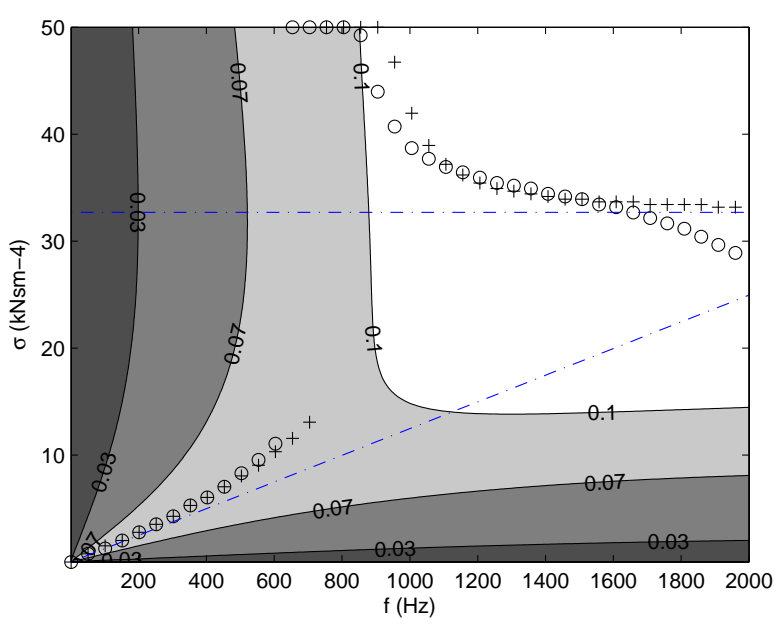

(c) $\phi_{p}=0.75$

FIG. 5. Influence of $\sigma$ on the attenuation rate: isovalues of $\mathfrak{I m} k_{d p} / k_{0}$ (- ) for $\phi_{p}=0.25,0.5$ and 0.75 . The markers stand for the maximum (with respect to $\sigma$ ) of the attenuation rate given by the DP model $\left(\max \mathfrak{I m} k_{d p} / k_{0}:+\right)$ and by the reference $\operatorname{model}^{7}\left(\max \mathfrak{I m} \beta / k_{0}: \circ\right)$. The optimal values given by Eqs.(35) and (38) are represented by dotted-dashed lines (-. ). Silencer with $H=0.2 \mathrm{~m}$ and one baffle.

A homogenization method for splitter silencer 


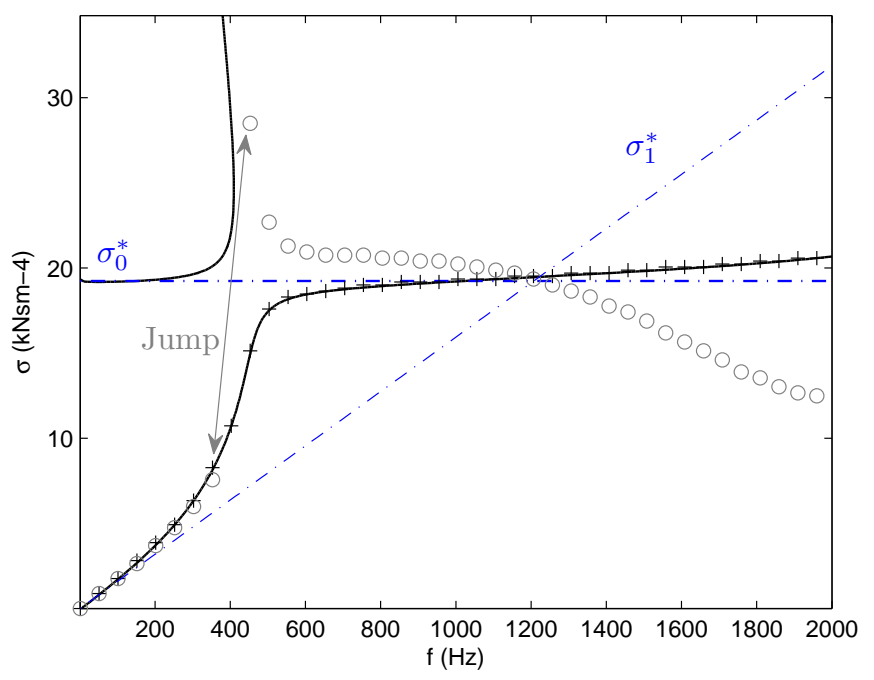

FIG. 6. Root locus of $\partial_{\sigma}\left(\mathfrak{I m} k_{d p}(\omega)\right)=0$ ( $\quad$ ) computed with finite difference. The makers stand for the maximum of the attenuation rate given by the DP model ( $\max \mathfrak{I m} k_{d p} / k_{0}:+$ ) and a reference solution mode computation $^{7}\left(\max \mathfrak{I m} \beta / k_{0}: \circ\right)$. The optimal values given by Eqs.(35) and (38) are represented by dotted-dashed lines $(-\cdot)$. Silencer with $H=0.2 \mathrm{~m}, \phi_{p}=0.5$ and one baffle.

TABLE III. Optimal resistivity value for various configurations.

\begin{tabular}{cccccc}
\hline \hline$\phi_{p}$ & $H$ & $N$ & $\left(r_{\Lambda}, c, \alpha_{\infty}, \phi_{m}\right)$ & $\begin{array}{c}\sigma_{0}^{*} \\
{\left[\mathrm{Nm}^{-4} \mathrm{~s}\right]}\end{array}$ & $\begin{array}{c}f_{d}^{*}=\frac{\omega_{d}^{*}}{2 \pi} \\
{[\mathrm{Hz}]}\end{array}$ \\
\hline- & {$[\mathrm{m}]$} & - & based on & 17375 & 518 \\
0.25 & 0.2 & 1 & $\mathrm{GW} 1$ & 19238 & 1054 \\
0.50 & 0.2 & 1 & $\mathrm{GW} 1$ & 32683 & 2483 \\
0.75 & 0.2 & 1 & $\mathrm{GW} 1$ & 9338 & 709 \\
0.75 & 0.7 & 1 & $\mathrm{GW} 1$ & 5497 & 301 \\
0.50 & 0.7 & 1 & $\mathrm{GW1}$ & 16490 & 904 \\
0.50 & 0.7 & 3 & $\mathrm{GW1}$ & 16404 \\
\hline \hline
\end{tabular}

By performing intensive numerical tests involving different silencer geometries and porous materials with low to moderate tortuosity (between 1 and 1.5), it was found that (35) provides a very good estimation of the optimal resistivity, except in the very low frequency regime. For illustrative purposes, Table III gives the values of $\sigma_{0}^{*}$ for various configurations. The latter correspond to available realistic materials. The last column gives the corresponding diffusion frequency $\omega_{d}^{*}=3 P_{0} /\left(b^{2} \sigma_{0}^{*} \phi_{m}\right)$. At this frequency the two predictions Eqs. (35) and (38) are nearly equal and the attenuation rate reaches its maximum value, as confirmed in Figs. 5.

\section{Practical examples}

In order to illustrate the practical use of the optimal value for the resistivity $\sigma_{0}^{*}$, we consider a typical baffle silencer used in ventilation systems ${ }^{4}$ with $L_{s}=1.2$ $\mathrm{m}$ and $H=0.7 \mathrm{~m}$. The silencer comprises $N=1$ or $N=3$ baffles. The parameters of the porous material are based on those of the GW1 wool $\left(r_{\Lambda}, c, \alpha_{\infty}, \phi_{m}\right)$ except that the resistivity can be modified independently. Fig. 7 shows the Transmission Losses of the silencer for three scenarios. The results were computed with the reference model $^{7}$ and thus can be considered as exact. In particular they take into account reflection mechanisms which are ignored in the derivation of the optimal resistivity. Note that the Mechel selection rule from (5) was used to identify the plane wave regime in order to set the frequency range of interest accordingly (around $500 \mathrm{~Hz}$ for $N=1$ and $1500 \mathrm{~Hz}$ for $N=3$ ). The performances of the silencer can be changed significantly by varying the resistivity from $\sigma_{0}^{*} / 10$ to $10 \sigma_{0}^{*}$ artificially. It is noteworthy that in each case, choosing $\sigma=\sigma_{0}^{*}$ yields the best attenuation. As expected, this choice is not optimal at low frequencies but the drop in performance is rather marginal. Finally, the DP model, which is an approximate and homogenized representation of the real silencer, is used in conjunction with the TMM in order to obtain an estimate of the TL for $\sigma=\sigma_{0}^{*}$. The curve shows somewhat good agreement, especially at low frequencies, and deviates strongly once the conditions for the plane wave regime in the silencer are no longer satisfied.

Previous results are valid only in the plane wave regime, assuming and incident plane wave with no mode conversion. However, it is interesting to test the validity of the optimal resistivity when higher mode are impinging the silencer. In this regard, two standard excitations are usually adopted ${ }^{23}$ : i) one assumes equal energy per mode or ii) equal energy density per mode (in this case the amplitudes of normalized propagating modes are all equal). Simulation with equal energy density per propagating mode are performed with a reference method on configurations (b) and (c) taken from Fig. 7. It is shown in Fig. 8 that, even with equal energy density per mode excitation, the optimal resistivity given in Eq. (35) provides the best compromise over a large frequency interval and allows achieving nearly the best attenuation as long as there is no modal conversion among incident modes, i.e. when the frequency is below the cut-off frequency given by the selection rule. For instance around $500 \mathrm{~Hz}$ for $N=1$ in Fig. 8a and around $1500 \mathrm{~Hz}$ for $N=3$ in Fig. 8b. Note that other calculations assuming equal energy per mode excitation leads to very similar results.

\section{CONCLUSION}

In this paper a double porosity model was used to predict and optimize the acoustic performances of baffletype silencers in ducts. The model, originally developed to describe the wave propagation in porous materials with slit-like perforations, is based on a homogenization process and thus yields an explicit expression for the effective wavenumber in the silencer. Through numerical comparisons, it was shown that the theory provides a good estimate as long as the pressure wave in the silencer propagates like a plane wave parallel to the duct axis.

In the last section of the paper, the analytical formula for the wavenumber was fully exploited to predict the conditions under which the best acoustic performances 


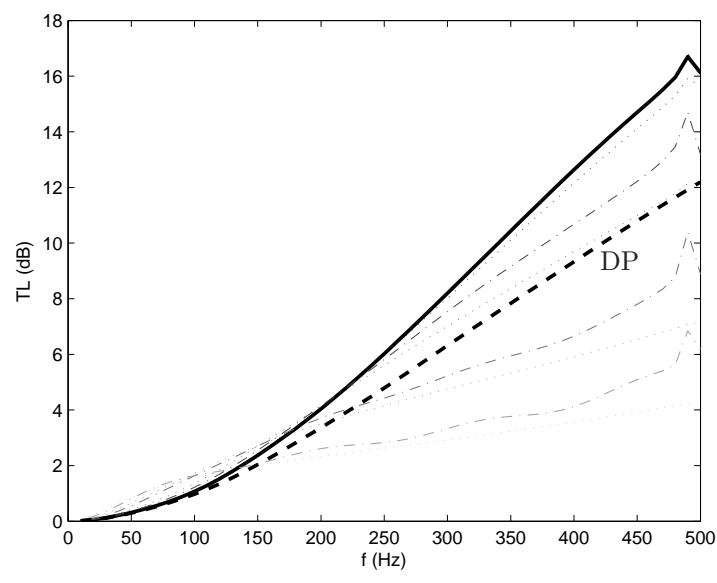

(a) $\phi_{p}=0.75, N=1$

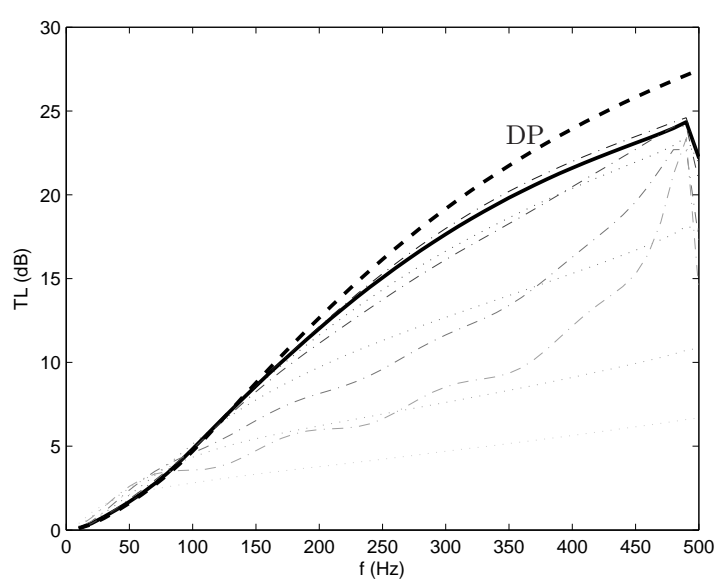

(b) $\phi_{p}=0.5, N=1$

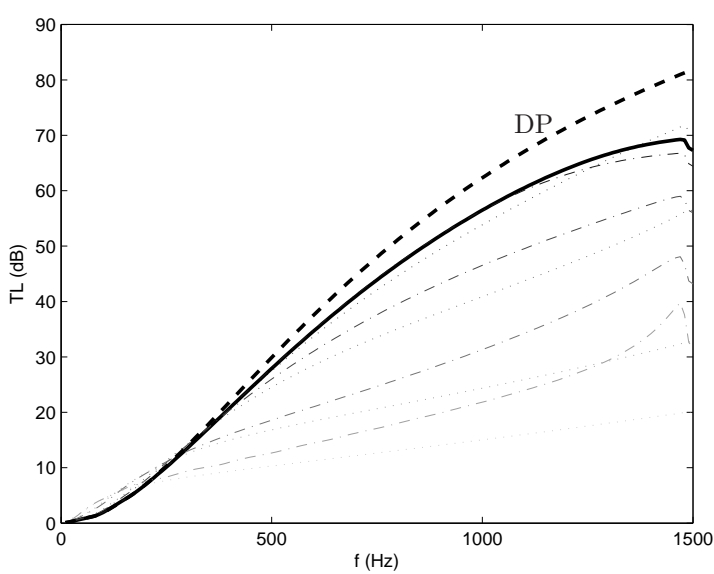

(c) $\phi_{p}=0.5, N=3$

FIG. 7. Transmission Loss evolution for $\sigma \in$ $\left[0.1 \sigma_{0}^{*}, \ldots, \sigma_{0}^{*}, \ldots, 10 \sigma_{0}^{*}\right]$ computed with a reference $\operatorname{method}^{7}$ for a plane wave excitation. If $\sigma<\sigma_{0}^{*}$, dotted line are used ( . . ). If $\sigma>\sigma_{0}^{*}$, dash-dotted line are used (-.-). The grayscale tends to the darkest value when the resistivity tends to $\sigma_{0}^{*}$. If $\sigma=\sigma_{0}^{*}$, black is used. The bold continuous line (- ) stands for the reference solution and the dashed line (- - ) stands for the DP model with TMM for $\sigma=\sigma_{0}^{*}$. Silencer with $H=0.70 \mathrm{~m}$ and $L_{s}=1.20 \mathrm{~m}$, based on wool GW1.

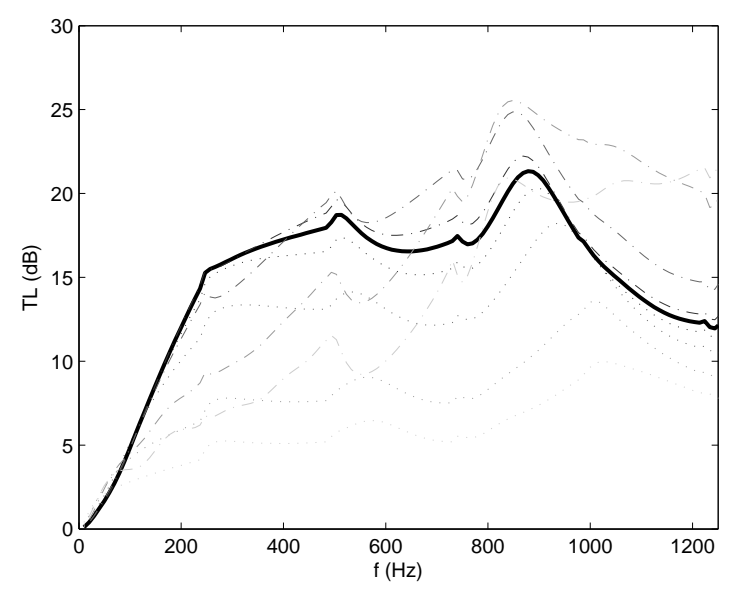

(a) $\phi_{p}=0.5, N=1$

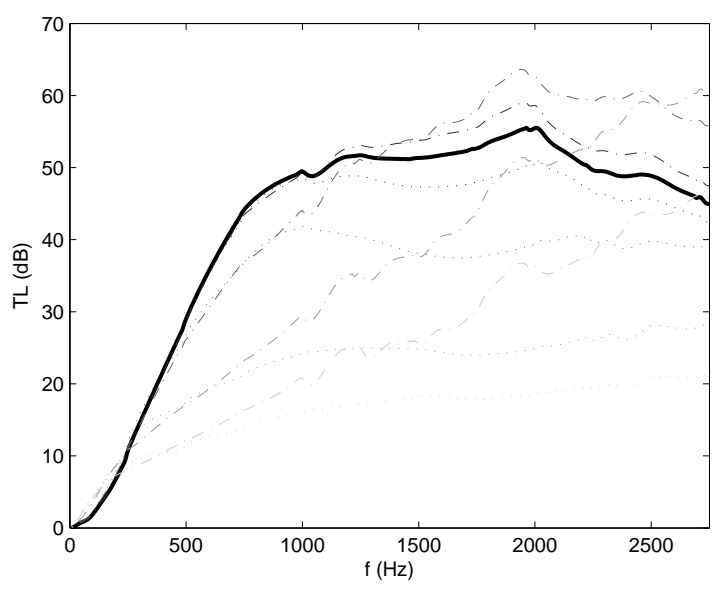

(b) $\phi_{p}=0.5, N=3$

FIG. 8. Transmission Loss evolution for $\sigma \in$ $\left[0.1 \sigma_{0}^{*}, \ldots, \sigma_{0}^{*}, \ldots, 10 \sigma_{0}^{*}\right]$ computed with a reference method $^{7}$ with Equal energy density per mode excitation case $^{23}$. Silencer with $H=0.70 \mathrm{~m}$ and $L_{s}=1.20 \mathrm{~m}$, based on wool GW1. Same legend as for Fig. 7

can be obtained. It turned out that: (i) at low frequency, the optimal resistivity given by (38) should vary linearly with the frequency; (ii) above a certain frequency, the value for the resistivity in Eq. (35) should be used. In practice, numerical predictions in terms of Transmission Losses show that Eq. (35) is always nearly optimal and that the drop in performance at low frequencies remains marginal.

In principle, the value proposed for the optimal resistivity is restricted to baffle-type silencers but there are good reasons to believe that a similar approach could be applied to optimize other types of silencers comprising a succession of porous layers such as expansion chambers filled with a porous material. It could also be used to design surface acoustic absorbers illuminated at normal incidence, in the spirit of Ref. 12 for instance. The present study focused on rigid frame materials and it would be interesting to investigate the behavior of limp materials ${ }^{22}$ and poroelastic materials. Convection effects 
due to the presence of mean flow could also be the subject for further investigation.

\section{ACKNOWLEDGEMENTS}

The authors would like to thank their industrial partners Alhyange Acoustique

(see http://www . alhyange.com/). This work was partly funded by the ANR Project METAUDIBLE No. ANR13-BS09-0003-01 funded jointly by ANR and FRAE.

\section{APPENDIX A: SELECTION RULE FOR BAFFLE-TYPE SILENCERS}

A complete explanation can be found in Ref. ${ }^{1}$. Here we shall recall the main ingredients of the theory. The idea is to view the silencer as an infinite succession of baffles (or equivalently, an infinite repetition of the duct interval $y \in[0, H])$. An incident plane wave of the form

$$
p^{i n c}=\exp \left(\mathrm{i}\left(\alpha^{i n c} y-\beta z\right)\right)
$$

gives rise to a series of reflected plane waves

$$
p=\sum_{q \in \mathbb{Z}} R_{q} \exp \left(\mathrm{i}\left(\alpha_{q} y+\beta_{q} z\right)\right)
$$

The periodic nature (with period $d=H / N$ ) of the problem implies the Floquet relation

$$
\alpha_{q}=\alpha^{i n c}+\frac{2 \pi q}{d} .
$$

In the context of guided waves in a symmetric silencer, a duct mode is simply the sum of two plane waves since

$$
\begin{aligned}
2 \cos \left(\alpha_{q} y\right) \exp \left(-\mathrm{i} \beta_{q} z\right)= & \exp \left(\mathrm{i}\left(\alpha_{q} y-\beta_{q} z\right)\right) \\
& +\exp \left(\mathrm{i}\left(-\alpha_{q} y-\beta_{q} z\right)\right)
\end{aligned}
$$

where $\alpha_{q}$ must correspond to the transverse wavenumber of a duct mode, i.e. $\alpha_{q}=n \pi / H$. The Floquet relation becomes:

$$
n=n^{i n c}+2 q N,
$$

which is the selection rule given in Ref. ${ }^{1}$ which reflects the two periodicity scales $H$ and $d$. Equation (A5) holds for all scattered modes including the transmitted ones.

\section{APPENDIX B: RIGID FRAME MODEL}

Porous materials with rigid skeleton were well described by the Johnson-Champoux-Allard (JCA) equivalent fluid model ${ }^{17}$ (Chap. 5). This equivalent fluid has the equivalent density $\left(\mathrm{e}^{+\mathrm{i} \omega t}\right)$

$$
\rho_{m}=\frac{\alpha_{\infty} \rho_{0}}{\phi_{m}}\left[1-\mathrm{i} \frac{\omega_{b}}{\omega} G_{J}(\omega)\right]
$$

and the equivalent bulk modulus,

$$
K_{m}=\frac{\gamma P_{0} / \phi_{m}}{\gamma-(\gamma-1)\left[1-\mathrm{i} c^{2} r_{\Lambda}^{2} \frac{\omega_{b}}{\operatorname{Pr} \omega}\left(1+\frac{\mathrm{i}}{2 c^{2} r_{\Lambda}^{2}} \frac{\operatorname{Pr} \omega}{\omega_{b}}\right)^{1 / 2}\right]^{-1}} .
$$

Here, $G_{J}(\omega)=\sqrt{1+\frac{\mathrm{i} c^{2}}{2} \frac{\omega}{\omega_{b}}}, \phi_{m}$ is the porosity, $\sigma$ is the flow resistivity, $\Lambda$ is the viscous length, $\Lambda^{\prime}$ is the thermal length and $\alpha_{\infty}$ is the tortuosity. To express $G_{J}$ as a function of $\omega_{b}$, the expression

$$
\Lambda^{2}=\frac{1}{c^{2}} \frac{8 \eta}{\omega_{b} \rho_{0}}
$$

given in Ref. 17 (Eq. (5.25)) is used. The coefficient $c$, is the square root of the shape factor and is close to unity and $c \in[0.8,1.1]$ (see ${ }^{17}$ (Tab. 4.1, p. 64)). The ratio between both characteristic length $r_{\Lambda}=\Lambda / \Lambda^{\prime}$ is introduced and ranges generally between 0.5 and 0.33 . The parameters used in this paper are given in Tab. I.

Moreover, $\gamma$ is the air specific heat ratio and $P_{0}$ is the atmospheric pressure, $\operatorname{Pr}$ is the Prandtl number and $\eta$ is the dynamic viscosity and $\kappa$ is the thermal conductivity of air. If $\omega \ll \omega_{b}$, viscous forces are dominant and the effective parameters can be replaced by the low-frequency approximations (approximated quantities are denoted by symbol " "):

$$
\tilde{\rho}_{m}=\rho_{0} \frac{\alpha_{\infty}}{\phi_{m}}\left(r-\mathrm{i} \frac{\omega_{b}}{\omega}\right),
$$

with $r=1+c^{2} / 4$ and

$$
\tilde{K}_{m}=\frac{P_{0}}{\phi_{m}}\left(1+\mathrm{i} \Upsilon \frac{\omega}{\omega_{b}}\right),
$$

where

$$
\Upsilon=\frac{\gamma-1}{\gamma} \frac{\operatorname{Pr}}{\left(r_{\Lambda} c\right)^{2}}
$$

In practice, $\Upsilon \in[1.25,1.48]$.

${ }^{1}$ F. P. Mechel, "Theory of baffle-type silencers", Acustica 70, 93-111 (1990).

${ }^{2}$ K. Tam and F. Fahy, "A theoretical and experimental investigation of sound intensity distribution within a splitter silencer", J. Sound Vib. 151, 213-246 (1991).

3 R. Kirby, "Simplified techniques for predicting the transmission loss of a circular dissipative silencer", J. Sound Vib. 243, 403-426 (2001).

${ }^{4}$ R. Kirby, "The influence of baffle fairings on the acoustic performance of rectangular splitter silencers", J. Acoust. Soc. Am. 118, 2302-2312 (2005).

5 J. B. Lawrie and R. Kirby, "Mode-matching without rootfinding: Application to a dissipative silencer", J. Acoust. Soc. Am. 119, 2050-2061 (2006).

6 B. Nennig, E. Perrey-Debain, and M. Ben Tahar, "A mode matching method for modelling dissipative silencers lined with poroelastic materials and containing mean flow", J. Acoust. Soc. Am. 128, 3308-3320 (2010).

7 R. Binois, E. Perrey-Debain, N. Dauchez, B. Nennig, J.-M. Ville, and G. Beillard, "On the efficiency of parallel baffletype silencers in rectangular ducts: prediction and measurement", Acta Acust. United Ac. In Press, 11 (2014). 
${ }^{8}$ R. Kirby, "A comparison between analytic and numerical methods for modelling automotive dissipative silencers with mean flow", J. Sound Vib. 325, 565-582 (2009).

${ }^{9}$ B. Nennig, M. Ben Tahar, and E. Perrey-Debain, "A displacement-pressure finite element formulation for analyzing the sound transmission in ducted shear flows with finite poroelastic lining", J. Acoust. Soc. Am. 130, 42-51 (2011).

10 J.-L. Auriault and C. Boutin, "Deformable media with double porosity- III: Acoustics", Transp. Porous Media 14, 143-162 (1994).

$11 \mathrm{X}$. Olny and C. Boutin, "Acoustic wave propagation in double porosity media", J. Acoust. Soc. Am. 114, 73-89 (2005).

12 F. C. Sgard, X. Olny, N. Atalla, and F. Castel, "On the use of perforations to improve the sound absorption of porous materials", Appl. Acoust. 66, 625-651 (2005).

13 F.-X. Bécot, L. Jaouen, and E. Gourdon, "Application of the dual porosity theory to irregularly shaped porous materials", Acta Acust. 94, 715-724 (2008).

14 O. Dazel, F.-X. Bécot, and L. Jaouen, "Biot effects for sound absorbing double porosity materials", Acta Acust. United Ac. 98, 567-576 (2012).

15 R. Venegas and O. Umnova, "Acoustical properties of double porosity granular materials", J. Acoust. Soc. Am. 130, 2765-2776 (2011).

16 E. Gourdon and M. Seppi, "On the use of porous inclu- sions to improve the acoustical response of porous materials: Analytical model and experimental verification", Appl. Acoust. 71, 283-298 (2010).

17 J.-F. Allard and N. Atalla, Propagation of Sound in Porous Media: Modeling Sound Absorbing Materials (second edition), 372pp (John Wiley \& Sons, Chichester) (2009).

18 M. L. Munjal, Acoustics of Ducts and Mufflers, 352pp (Wiley, New York) (1987).

19 D. Homentcovschi and R. N. Miles, "A re-expansion method for determining the acoustical impedance and the scattering matrix for the waveguide discontinuity problem", J. Acoust. Soc. Am. 128, 628-638 (2010).

${ }^{20}$ N. Atalla, R. Panneton, F. C. Sgard, and X. Olny, "Acoustic absorption of macro-perforated porous materials", J. Sound Vib. 243, 659-678 (2001).

21 D. Lafarge, P. Lemarinier, J.-F. Allard, and V. Tarnov, "Dynamic compressibility of air in porous structures at audible frequencies", J. Acoust. Soc. Am. 102, 1995-2006 (1997).

22 O. Doutres, N. Dauchez, J.-M. Génevaux, and O. Dazel, "Validity of the limp model for porous materials: a criterion based on the Biot theory", J. Acoust. Soc. Am. 122, 2038-2048 (2007).

23 P. Joseph, C. Morfey, and C. Lowis, "Multi-mode sound transmission in ducts with flow", Journal of Sound and Vibration 264, 523-544 (2003). 